\title{
Agreement of Ultra-Short-Term Heart Rate Variability Recordings During Overseas Training Camps in Under-20 National Futsal Players
}

\author{
Yung-Sheng Chen ${ }^{1,2}$, Jeffrey C. Pagaduan ${ }^{3}$, Pedro Bezerra ${ }^{4,5}$, \\ Zachary J. Crowley-McHattan ${ }^{6}$, Cheng-Deng Kuo ${ }^{7,8,9 *}$ and Filipe Manuel Clemente ${ }^{4,10 *}$ \\ ${ }^{1}$ Department of Exercise and Health Sciences, University of Taipei, Taipei, Taiwan, ${ }^{2}$ Exercise and Health Promotion \\ Association, New Taipei City, Taiwan, ${ }^{3}$ College of Health and Medicine, School of Health Sciences, University of Tasmania, \\ Hobart, TAS, Australia, ${ }^{4}$ Escola Superior Desporto e Lazer, Instituto Politécnico de Viana do Castelo, Viana do Castelo, \\ Portugal, ${ }^{5}$ The Research Centre in Sports Sciences, Health Sciences and Human Development, Vila Real, Portugal, ${ }^{6}$ School \\ of Health and Human Sciences, Southern Cross University, Lismore, NSW, Australia, ${ }^{7}$ Department of Medical Research, \\ Taipei Veterans General Hospital, Taipei, Taiwan, ${ }^{8}$ Department of Medicine, Taian Hospital, Taipei, Taiwan, ${ }^{9}$ Tanyu Research \\ Laboratory, Taipei, Taiwan, ${ }^{10}$ Instituto de Telecomunicações, Delegação da Covilhã, Lisboa, Portugal
}

\section{OPEN ACCESS}

Edited by:

Fábio Yuzo Nakamura, Instituto Universitário da Maia (ISMAI), Portugal

Reviewed by:

Andrew A. Flatt, Georgia Southern University, United States

Cesar Cavinato Cal Abad, Faculdade Lusófona de São Paulo, Brazi

*Correspondence: Cheng-Deng Kuo cdkuo23@gmail.com

Filipe Manuel Clemente filipe.clemente5@gmail.com

Specialty section: This article was submitted to Movement Science and Sport

Psychology,

a section of the journal

Frontiers in Psychology

Received: 26 October 2020 Accepted: 12 January 2021 Published: 05 February 2021

Citation: Chen Y-S, Pagaduan JC, Bezerra P, Crowley-McHattan ZJ, Kuo C-D and Clemente FM (2021) Agreement of Ultra-Short-Term Heart Rate Variability Recordings During Overseas Training Camps in Under-20 National Futsal

Players. Front. Psychol. 12:621399. doi: 10.3389/fpsyg.2021.621399
Background: Monitoring the daily change in resting heart rate variability (HRV) can provide information regarding training adaptation and recovery status of the autonomic nervous system (ANS) during training camps. However, it remains unclear whether postural stabilization is essential for valid and reliable ultra-short-term (HRV UST) recordings in short-term overseas training camps.

Design: Observational and longitudinal study.

Purpose: This study aimed to investigate ultra-short-term heart rate variability recordings under stabilization or post-stabilization periods in four overseas training camps.

Participant: Twenty-seven U-20 male national team futsal players voluntarily participated in this study.

Method: Resting HRV was evaluated for 10 min during the early morning of each training camp. The natural logarithm of the root mean square of successive normal-to-normal interval differences (LnRMSSD) was used for comparisons. Time segments of HRV were divided into two periods with three measures within each: (1) the first 30-s (1st_30 s LnRMSSD), the first 60-s (1st_60 s LnRMSSD), and the 5-min standard (1st_5 min LnRMSSD) during stabilization; (2) the first 30-s (2nd_30 s LnRMSSD), the first 60-s (2nd_60 s LnRMSSD), and the 5-min standard (2nd_5 min LnRMSSD) after stabilization.

Result: The results demonstrated trivial to small ES (-0.03; 0.46), very large to nearly perfect ICC $(0.76$; 0.98$)$, and narrow range of SEM $(0.06 ; 0.31)$ when all time segments of HRV UST were compared to the 1st_5 min and 2nd_5 min HRV. Furthermore, the magnitude of the correlation coefficients ranged from very high to nearly perfect for all the time segments $(r=0.83 ; 0.97)$. The HRV UST posted excellent agreement in all time segments (bias $=-0.05 ; 0.12$ ) with/without postural stabilization. Trivial to small levels of effect size in all time segments of LnRMSSD mean $(0.02 ; 0.41 \mathrm{ES})$ and $\operatorname{LnRMSSD}_{\mathrm{CV}}(-0.49 ;-0.02 \mathrm{ES})$ across overseas training camps was identified. 
Conclusion: The first 30 or 60-s LnRMSSD recordings can be used to evaluate daily cardiac-autonomic function during overseas training camps in futsal players. The process for stabilization seems to be unnecessary for measuring the morning resting LnRMSSD in overseas training camps among young adult futsal players.

\section{Keywords: heart rate variability, autonomic nervous system, overseas training camps, futsal training, ultra-short- term recording}

\section{INTRODUCTION}

Heart rate (HR) variability (HRV) is a neurophysiological marker that reflects the cardiac-related activation regulated by the autonomic nervous system (ANS) (Buchheit, 2014). Assessment of HRV requires recording a time series of $\mathrm{HR}$ beat-to-beat intervals ( $R$ wave-to- $R$ wave interval, $R R I$ ) via a non-invasive electrocardiographic device (ECG) or a HR monitor sensor. The measurement of HRV can help sports practitioners and coaches interpret autonomic function in terms of training adaptations (i.e. increase in RRI is associated with improvement of aerobic capacity) (Sandercock et al., 2005; Plews et al., 2013a), recovery status (Buchheit et al., 2007; Nakamura et al., 2009; Chen et al., 2019), and autonomic health during international competitions (Flatt and Howells, 2019; Flatt et al., 2019; Muñoz-López et al., in press).

The standard process to collect HRV data requires a 5-min short-term recording period preceded by a 5 -min stabilization period (Task Force of the European Society of Cardiology the North American Society of Pacing Electrophysiology., 1996). However, this assessment method is limited to specific circumstances, i.e., during traditional training schedules, due to the time-consuming nature of this type of assessment. Yet, with recent methodological developments, the assessment of ultrashort-term HRV (HRV UST), HRV recording of $<60$ s, have been established to improve the usability of this technique in fieldbased settings (Castaldo et al., 2019).

In a practical sense, HRV UST assessment during sports training can be used to understand cardiac modulation during exercise or physiological adaption after an acute and chronic training regime. Nakamura et al. (2015) reported excellent limits of agreement and acceptance in 1-min HRV UST (natural logarithm of the root mean square of successive normal-tonormal interval differences, LnRMSSD UST), compared to a criterion of 5-min LnRMSSD (a 5-min HRV record after 5-min stabilization). The correlation between changes in LnRMSSD UST (i.e., $0-1 ; 1-2 ; 2-3 ; 3-4 ; 4-5 \mathrm{~min}$ ) and LnRMSSD criterion was between 0.45 and 0.75 , with the highest value $(r=0.75$; 90\% CI: $0.55-0.85)$ found between LnRMSSD UST $_{\text {at }} 1-2 \mathrm{~min}$ and LnRMSSD criterion, indicating measurement validity and agreement of HRV UST assessment after 1-min of stabilization. In addition, Krejčí et al. (2018) compared LnRMSSDUST every $30 \mathrm{~s}$ in 5-min stabilization records to a 5 -min criterion $(6-10 \mathrm{~min})$ in 30 endurance athletes at the national level ( 10 ski runners, 8 road cyclists, and 12 cross-country skiers) and 30 university students. When LnRMSSD $\mathrm{UST}_{\mathrm{U}}$ and HR were measured in the supine position, the minimal stabilization period to stabilize both indices was 60-s for endurance athletes and 90-s for university students for valid and reliable estimations. Interestingly, Pereira et al. (2016) demonstrated excellent reliability and limits of agreement of 1-min HRV UST during 5-min stabilization in 35 elite futsal players. An additional observation in an athletic population reported by Flatt and Esco (2016) also supports this notion. However, interpretation of these findings may be limited due to the heterogeneity of the sample pools from mixed genders and training regimens. Despite the time efficiency of data collection using HRV $_{\text {UST }}$, there are no standard recommendations for using HRV UST with or without stabilization.

Monitoring the daily change in resting HRV can provide information regarding training adaptation and recovery status of ANS health during national team training camps (Flatt and Howells, 2019; Flatt et al., 2019). During a national team training camp, soccer players who played more than $60 \mathrm{~min}$ of match play experienced augmented ANS modulation (by measuring daily morning LnRMSSD) after a friendly match. This profound effect lasting for $72 \mathrm{~h}$ after (Muñoz-López et al., in press). Thus, it is important to monitor the HRV parameters for assessing recovery status during training camps. It was recently observed that 30-s mean value of LnRMSSD (LnRMSSD mean) measure was accurate and valid compared to the standard 5-min LnRMSSD measure during both training camps (domestic and overseas) and international competition in a male U-20 national futsal team. In contrast, coefficient of variation of LnRMSSD (LnRMSSD ${ }_{c v}$ ) measure required at least $2 \mathrm{~min}$ recordings to provide valid and reliable HRV measures during training camps (Chen et al., 2020; Clemente F. M et al., 2020). However, the interpretation of these findings were highlighted as being after 5-min postural stabilization period.

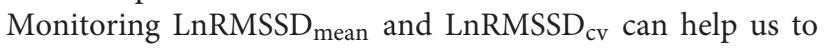
understand the vagal-related adaptation and psychophysiological status associated with physical fitness during training period (i.e., increase in $\operatorname{LnRMSSD}_{\text {mean }}$, decrease in $\operatorname{LnRMSSD}_{\mathrm{cv}}$, and improvement of aerobic capacity) (Nakamura et al., 2020). Most of previous studies reported agreement of HRV UST based on crossectional designs, in this study, weekly HRV UST of LnRMSSD $_{\text {mean }}$ and $\operatorname{LnRMSSD}_{\mathrm{cv}}$ was investigated during four overseas training camp in a male U-20 national futsal team. In light of the abovementioned studies, the purpose of this study was twofold. Firstly, to compare HRV UST recordings within stabilization and after stabilization periods during short-term overseas training camps in U-20 national futsal players as a surrogate to the traditional 5-min standard measures. Secondly, to compare the variation of HRV UST measures among shortterm overseas training camps. It was hypothesized that HRV UST 
recordings during stabilization and after stabilization would show similar degrees of agreement and reproducibility to 5-min HRV records. The secondary hypothesis was that $\mathrm{HRV}_{\text {UST }}$ would show similar characteristics among overseas training camps.

\section{MATERIALS AND METHODS}

\section{Experimental Approach to the Problem}

This study was a cross-sectional and observational study. Morning resting HRV was measured in four overseas training camps prior to the Asian U-20 Futsal Championship final. The HRV UST assessment during the first 5-min (stabilization) and second 5-min (after stabilization) was compared. The time segments of HRV records were divided into the first 30-s (1st_30 s LnRMSSD), the first 60-s (1st_60 s LnRMSSD), and the 5-min recording (1st_5 min LnRMSSD) during stabilization, and the first 30-s (2nd_30 s LnRMSSD), the first 60-s (2nd_60 s LnRMSSD), and the 5-min recording (2nd_5 min LnRMSSD) after stabilization.

The number of participating players varied from camp to camp due to budgeting and logistical issues (1st training camp: 6 days, 15 players; 2nd training camp: 5 days, 20 players; 3rd training camp: 6 days, 17 players; 4th training camp: 10 days, 14 players). The players involved in the fourth oversea training camp were the final registered players for the continental tournament. All assessments were conducted during the overseas training camps prior to the 2019 Asian Football Confederation U-20 Futsal Championship Final (Tabriz, Iran). Table 1 shows detailed information regarding the schedule of four overseas training camps.

\section{Experimental Procedure}

During each oversea training camp, the players were lodged in a domestic hotel one night before international travel. All players undertook daily resting HRV measures before breakfast in the early morning during the training camps. The players were instructed to maintain a comfortable sitting position for resting HRV assessment. The players were informed to control their breathing with their preferred patterns while their eyes were closed. The morning resting HRV was recorded via an individual portable Polar HR monitor (Polar team Pro, Polar Electro, Kemple, Finland). All sensors were synced to a Polar team pro dock. All data were uploaded to a secure cloud server and then subsequently exported to a laptop for data analysis. The duration of the resting HRV assessment was 10-min. All measures were performed in a quiet and spacious meeting room between 7 a.m. and 8 a.m. in local time.

\section{Participants}

Twenty-four outfield players and three goalkeepers, male futsal players were recruited and voluntarily participated this study from Chinese Taipei U-20 national futsal team (mean \pm standard deviation: age $=17.93 \pm 0.87$ yrs; height $=1.71 \pm 0.07 \mathrm{~m}$; body weight $=65.39 \pm 9.39 \mathrm{~kg}$; body fat $=12.54 \pm 2.76 \%$; maximal aerobic capacity $=51.98 \pm 3.07 \mathrm{ml} \cdot \mathrm{kg}^{-1} \cdot \mathrm{min}^{-1}$ ). The players signed informed consent forms and were all familiarized with experimental procedures prior to participation. The study was approved by the Human Ethics Committee of the University of Taipei (UT-IRB-2018-068) and undertaken in accordance with the Declaration of Helsinki and its later amendments in 2013.

\section{Heart Rate Variability}

A portable telemetric HR monitor system was used to record the resting HRV (Polar team Pro, Polar Electro, Kemple, Finland). Each player was issued an HR sensor and HR strap for the entire duration of the training camp. Kubios HRV analysis software (Premium version 3.2.0., Kubios, Kuopio, Finland) was used to calculate LnRMSSD. Artifact correction was set at a medium threshold level, and the window width was set at $300 \mathrm{~s}$ with a window overlap of $50 \%$. Smoothing priors set at 500 Lambda were used for detrending methods (Tarvainen et al., 2014). If the percentage of ectopic beats in daily measure were $>5 \%$, then the data was excluded from the analysis.

\section{Statistical Analyses}

Descriptive data of the measured variables are presented as LnRMSSD $_{\text {mean }}$ or LnRMSSD $_{\mathrm{cv}}$ and standard deviation (SD). Inter-differences of HRV UST to standard values was analyzed by using Cohen's d effect size (ES). The level of ES was interpreted as trivial $(0.0-0.2)$, small $(0.2-0.6)$, moderate (0.61.2), large (1.2-2.0), very large (>2.0) (Hopkins et al., 2009). For reliability analysis, interclass correlation coefficients (ICC) with a two-way random model and a single measure were used to determine relative reliability. The level of ICC values were assessed as nearly perfect $(0.9-1)$, very large (0.70-89), large (0.50-69), moderate (0.31-49), and small (0-0.3) (Hopkins et al., 2009). Moreover, the standard error of measurement (SEM) was used to analyzed the absolute values of reliability. The SEM was calculated as SD $\sqrt{1-\text { ICC }}$ (Weir, 2005). For validity analysis, the relationship of HRV recordings between each time segment were assessed via Pearson product-moment correlation coefficient $(r)$. The magnitude of the correlation coefficients was determined as trivial $(r<0.1)$, small $(0.1<$ $r<0.3)$, moderate $(0.3<r<0.5)$, high $(0.5<r<0.7)$, very high $(0.7<r<0.9)$, nearly perfect $(r>0.9)$, and perfect $(r=1)$ (Hopkins et al., 2009). In addition, BlandAltman plots were used to evaluate the upper and lower limits of agreements among all time segments of LnRMSSD (Bland and Altman, 1986). Statistical analyses were conducted using by SPSS $^{\circledR}$ Statistics version 25.0 (IBM, Armonk, NY, USA) and Microsoft Excel 2016 (Microsoft Corporation, Redmond, WA, USA).

\section{RESULT}

\section{Agreement and Reliability of Ultra-Short-Term Heart Rate Variability}

The daily HRV recordings from all players were compliance with full attendance during the training camps. For LnRMSSD mean, small ES and very large ICC values were found between the $1 \mathrm{st} \_30 \mathrm{~s}$ and 1st_5 min, or $1 \mathrm{st} \_30 \mathrm{~s}$, and 2 nd_5 min in the 2nd training camp. A similar finding was reported in the 3rd training camp, except for the ICC comparison of the 1st_30 s and 1st_5 min. Moderate ES and very large ICC values 
TABLE 1 | The schedule of overseas training camps prior to the continental tournament final.

\begin{tabular}{|c|c|c|c|c|c|}
\hline Location & Date & $\begin{array}{l}\text { Duration } \\
\text { (days) }\end{array}$ & $\begin{array}{c}\text { Players } \\
\text { (numbers) }\end{array}$ & $\begin{array}{l}\text { Training sessions } \\
\text { (sessions) }\end{array}$ & $\begin{array}{l}\text { Friendly matches } \\
\text { (games) }\end{array}$ \\
\hline $\begin{array}{l}\text { 1st TC } \\
\text { Shenzhen, China }\end{array}$ & $\begin{array}{l}\text { July 28th-August 2nd } \\
2018\end{array}$ & 6 & 15 & 1 session (115-min) & $\begin{array}{l}4 \text { games }(89,100,101 \\
91-\min )\end{array}$ \\
\hline $\begin{array}{l}\text { 2nd TC } \\
\text { Nagoya, Japan }\end{array}$ & $\begin{array}{l}\text { November 19th-23th } \\
2018\end{array}$ & 5 & 20 & $\begin{array}{l}3 \text { sessions }(109,128,126, \\
76-\text { min, ) }\end{array}$ & 3 games $(97,75,77-\mathrm{min})$ \\
\hline $\begin{array}{l}\text { 3rd TC } \\
\text { Osaka, Japan }\end{array}$ & April 7th-12th 2019 & 6 & 17 & $\begin{array}{l}4 \text { sessions }(91,108,95 \\
117-\text { min) }\end{array}$ & $\begin{array}{l}4 \text { games }(107,101,102, \\
90 \text {-min) }\end{array}$ \\
\hline $\begin{array}{l}\text { 4th TC } \\
\text { Luso, Portugal }\end{array}$ & June 1st-10th 2019 & 10 & 14 & 3 sessions $(83,73,90-\mathrm{min})$ & $\begin{array}{l}5 \text { games }(87,82,78,83, \\
67 \text {-min) }\end{array}$ \\
\hline
\end{tabular}

TC, training camp.

TABLE 2 | Mean of natural logarithm of root mean square differences between adjacent normal R-R intervals (LnRMSSD) during 0-30 s, 0-60 s, and 0-5 min criterion in stabilization (1st_5 min) and after stabilization (2nd_5 min) assessments.

\begin{tabular}{|c|c|c|c|c|c|c|c|c|c|}
\hline \multirow[t]{2}{*}{ Training camp } & \multirow[t]{2}{*}{ Parameters } & \multicolumn{2}{|c|}{ ES $(90 \% \mathrm{Cl})$} & \multicolumn{2}{|c|}{ ICC (90\% CI) } & \multicolumn{2}{|c|}{ SEM } & \multicolumn{2}{|c|}{ Bias ( $\pm 1.96 * S D)$} \\
\hline & & 1st_5 min & 2nd_5 min & 1st_5 min & 2nd_5 min & 1st_5 min & 2nd_5 min & 1st_5 min & 2nd_5 min \\
\hline \multirow[t]{4}{*}{$\begin{array}{l}\text { 1st training camp } \\
(n=15)\end{array}$} & 1st_30s & $\begin{array}{c}-0.13 \\
(-0.73 ; 0.47)^{\star}\end{array}$ & $\begin{array}{c}-0.08 \\
(-0.69 ; 0.52)^{\star}\end{array}$ & $\begin{array}{c}0.87(0.93 \\
0.99)^{\ddagger}\end{array}$ & $\begin{array}{c}0.82(0.61 \\
0.92)^{\ddagger}\end{array}$ & 0.14 & 0.17 & $\begin{array}{c}-0.06 \\
(-0.52 ; 0.40)\end{array}$ & $\begin{array}{c}-0.05 \\
(-0.61 ; 0.52)\end{array}$ \\
\hline & 1st_60s & $\begin{array}{c}-0.14 \\
(-0.74 ; 0.46)^{\star}\end{array}$ & $\begin{array}{c}-0.10 \\
(-0.70 ; 0.50)^{\star}\end{array}$ & $\begin{array}{c}0.95(0.88 \\
0.98)^{\S}\end{array}$ & $\begin{array}{l}0.91(0.79 \\
0.96)^{\S}\end{array}$ & 0.10 & 0.14 & $\begin{array}{c}-0.06 \\
(-0.35 ; 0.23)\end{array}$ & $\begin{array}{c}-0.05 \\
(-0.47 ; 0.37)\end{array}$ \\
\hline & 2nd_30s & $\begin{array}{c}0.07(-0.53 \\
0.67)^{\star}\end{array}$ & $\begin{array}{c}0.10(-0.50 \\
0.70)^{\star}\end{array}$ & $\begin{array}{c}0.95(0.89 \\
0.98)^{\S}\end{array}$ & $\begin{array}{c}0.97(0.92 \\
0.99)^{\S}\end{array}$ & 0.14 & 0.11 & $\begin{array}{c}0.05(-0.31 \\
0.40)\end{array}$ & $\begin{array}{c}0.06(-0.22 \\
0.34)\end{array}$ \\
\hline & 2nd_60s & $\begin{array}{c}0.12(-0.48 \\
0.72)^{\star}\end{array}$ & $\begin{array}{c}0.15(-0.45 \\
0.75)^{\star}\end{array}$ & $\begin{array}{c}0.95(0.87 \\
0.98)^{\S}\end{array}$ & $\begin{array}{c}0.95(0.87 \\
0.99)^{\S}\end{array}$ & 0.14 & 0.14 & $\begin{array}{c}0.08(-0.30 \\
0.45)\end{array}$ & $\begin{array}{c}0.09(-0.25 \\
0.43)\end{array}$ \\
\hline \multirow[t]{4}{*}{$\begin{array}{l}\text { 2nd training camp } \\
(n=20)\end{array}$} & 1st_30s & $\begin{array}{c}0.24(-0.28 \\
0.77)^{\#}\end{array}$ & $\begin{array}{c}0.22(-0.39 \\
0.84)^{\#}\end{array}$ & $\begin{array}{c}0.86(0.69 \\
0.94)^{\ddagger}\end{array}$ & $\begin{array}{c}0.76(0.52 \\
0.89)^{\ddagger}\end{array}$ & 0.24 & 0.31 & $\begin{array}{c}0.16(-0.42 \\
0.73)\end{array}$ & $\begin{array}{c}0.20(-0.56 \\
0.96)\end{array}$ \\
\hline & 1st_60s & $\begin{array}{c}0.16(-0.36 \\
0.68)^{\star}\end{array}$ & $\begin{array}{c}0.11(-0.50 \\
0.73)^{\star}\end{array}$ & $\begin{array}{c}0.91(0.80 \\
0.96)^{\S}\end{array}$ & $\begin{array}{c}0.82(0.64 \\
0.91)^{\ddagger}\end{array}$ & 0.20 & 0.28 & $\begin{array}{c}0.11(-0.39 \\
0.61)\end{array}$ & $\begin{array}{c}0.15(-0.54 \\
0.85)\end{array}$ \\
\hline & 2nd_30s & $\begin{array}{c}0.06(-0.46 \\
0.59)^{\star}\end{array}$ & $\begin{array}{c}-0.07 \\
(-0.68 ; 0.54)^{\star}\end{array}$ & $\begin{array}{l}0.90(0.79 \\
0.95)^{\S}\end{array}$ & $\begin{array}{c}0.88(0.76 \\
0.94)^{\ddagger}\end{array}$ & 0.20 & 0.22 & $\begin{array}{c}0.04(-0.51 \\
0.59)\end{array}$ & $\begin{array}{c}0.09(-0.49 \\
0.67)\end{array}$ \\
\hline & 2nd_60s & $\begin{array}{c}-0.03 \\
(-0.49 ; 0.55)^{\star}\end{array}$ & $\begin{array}{c}0.11(-0.41 \\
0.63)^{\star}\end{array}$ & $\begin{array}{c}0.92(0.83 \\
0.96)^{\S}\end{array}$ & $\begin{array}{c}0.91(0.81 \\
0.96)^{\S}\end{array}$ & 0.18 & 0.19 & $\begin{array}{c}0.02(-0.47 \\
0.51)\end{array}$ & $\begin{array}{c}0.07(-0.44 \\
0.58)\end{array}$ \\
\hline \multirow[t]{4}{*}{$\begin{array}{l}\text { 3rd training camp } \\
(n=17)\end{array}$} & 1st_30s & $\begin{array}{c}0.22(-0.34 \\
0.79)^{\#}\end{array}$ & $\begin{array}{c}0.46(-0.11 \\
1.04)^{\#}\end{array}$ & $\begin{array}{c}0.93(0.76 \\
0.97)^{\S}\end{array}$ & $\begin{array}{c}0.84(0.19 ; \\
0.95)^{\ddagger}\end{array}$ & 0.12 & 0.18 & $\begin{array}{c}0.11(-0.19 \\
0.41)\end{array}$ & $\begin{array}{c}0.22(-0.09 \\
0.52)\end{array}$ \\
\hline & 1st_60s & $\begin{array}{c}0.12(-0.45 \\
0.68)^{\star}\end{array}$ & $\begin{array}{c}0.33(-0.24 \\
0.90)^{\#}\end{array}$ & $\begin{array}{c}0.98(0.94 \\
0.99)^{\S}\end{array}$ & $\begin{array}{c}0.90(0.52 \\
0.97)^{\S}\end{array}$ & 0.07 & 0.16 & $\begin{array}{c}0.05(-0.13 \\
0.24)\end{array}$ & $\begin{array}{c}0.16(-0.13 \\
0.45)\end{array}$ \\
\hline & 2nd_30s & $\begin{array}{c}-0.07 \\
(-0.64 ; 0.49)^{\star}\end{array}$ & $\begin{array}{c}0.11(-0.45 \\
0.68)^{\star}\end{array}$ & $\begin{array}{c}0.96(0.92 \\
0.98)^{\S}\end{array}$ & $\begin{array}{c}0.93(0.84 \\
0.97)^{\S}\end{array}$ & 0.12 & 0.16 & $\begin{array}{c}-0.04 \\
(-0.34 ; 0.25)\end{array}$ & $\begin{array}{c}0.06(-0.33 \\
0.45)\end{array}$ \\
\hline & 2nd_60s & $\begin{array}{c}-0.07(0.64 \\
0.49)^{\star}\end{array}$ & $\begin{array}{c}0.11(-0.45 \\
0.68)^{\star}\end{array}$ & $\begin{array}{c}0.98(0.94 \\
0.99)^{\S}\end{array}$ & $\begin{array}{c}0.95(0.88 \\
0.98)^{\S}\end{array}$ & 0.08 & 0.13 & $\begin{array}{c}-0.04 \\
(-0.27 ; 0.18)\end{array}$ & $\begin{array}{c}0.06(-0.25 \\
0.37)\end{array}$ \\
\hline \multirow[t]{4}{*}{$\begin{array}{l}\text { 4th training camp } \\
(n=14)\end{array}$} & 1st_30s & $\begin{array}{c}0.15(-0.47 \\
0.77)^{\star}\end{array}$ & $\begin{array}{c}0.17(-0.45 \\
0.80)^{\star}\end{array}$ & $\begin{array}{c}0.96(0.87 \\
0.99)^{\S}\end{array}$ & $\begin{array}{c}0.90(0.77 \\
0.96)^{\S}\end{array}$ & 0.08 & 0.13 & $\begin{array}{c}0.06(-0.13 \\
0.26)\end{array}$ & $\begin{array}{c}0.07(-0.25 \\
0.40)\end{array}$ \\
\hline & 1st_60s & $\begin{array}{c}0.12(-0.50 \\
0.74)^{\star}\end{array}$ & $\begin{array}{c}0.14(-0.48 \\
0.77)^{\star}\end{array}$ & $\begin{array}{c}0.98(0.93 \\
0.99)^{\S}\end{array}$ & $\begin{array}{l}0.94(0.85 \\
0.98)^{\S}\end{array}$ & 0.06 & 0.10 & $\begin{array}{c}0.05(-0.08 \\
0.18)\end{array}$ & $\begin{array}{c}0.06(-0.20 \\
0.32)\end{array}$ \\
\hline & 2nd_30s & $\begin{array}{c}0.24(-0.38 \\
0.87)^{\#}\end{array}$ & $\begin{array}{c}0.27(-0.35 \\
0.90)^{\#}\end{array}$ & $\begin{array}{c}0.85(0.64 \\
0.94)^{\ddagger}\end{array}$ & $\begin{array}{l}0.90(0.68 \\
0.96)^{\S}\end{array}$ & 0.16 & 0.13 & $\begin{array}{c}0.10(-0.31 \\
0.51)\end{array}$ & $\begin{array}{c}0.11(-0.18 \\
0.40)\end{array}$ \\
\hline & 2nd_60s & $\begin{array}{c}0.10(-0.52 \\
0.72)^{\star}\end{array}$ & $\begin{array}{c}0.12(-0.50 \\
0.75)^{\star}\end{array}$ & $\begin{array}{c}0.94(0.86 \\
0.98)^{\S}\end{array}$ & $\begin{array}{c}0.97(0.90 \\
0.97)^{\S}\end{array}$ & 0.10 & 0.07 & $\begin{array}{c}0.04(-0.22 \\
0.31)\end{array}$ & $\begin{array}{c}0.05(-0.10 ; \\
0.21)\end{array}$ \\
\hline
\end{tabular}

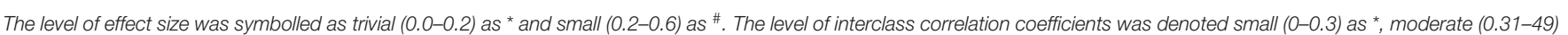

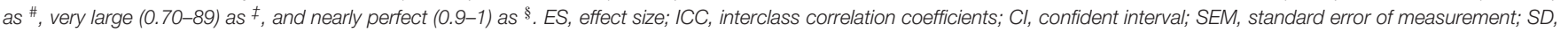
standard deviation; $n$, numbers.

were also found between the 2nd_30 s and 1st_5 min, or the 1st_30 s and 2nd_5 min. The other comparisons showed trivial ES and nearly perfect ICC values (Table 2). For LnRMSSD $\mathrm{cv}_{\mathrm{cv}}$, a wide range of ES $(-0.91 ;-0.02)$ and $\operatorname{ICC}(0.23 ; 0.88)$ values were found among the overseas training camps (Table 3). In Figure 1, the results of $\mathrm{LnRMSSD}_{\text {mean }}$ exhibited nearly perfect 
TABLE 3 | Coefficient of variation of natural logarithm of root mean square differences between adjacent normal R-R intervals (LnRMSSD) during 0-30 s, 0-60 s, and 0-5 min criterion in stabilization (1st_5min) and after stabilization (2nd_5min) assessments.

\begin{tabular}{|c|c|c|c|c|c|c|c|c|c|}
\hline \multirow[t]{2}{*}{ Training camp } & \multirow[t]{2}{*}{ Parameters } & \multicolumn{2}{|c|}{ ES $(90 \% \mathrm{Cl})$} & \multicolumn{2}{|c|}{ ICC $(90 \% \mathrm{Cl})$} & \multicolumn{2}{|c|}{ SEM } & \multicolumn{2}{|c|}{ Bias $( \pm 1.96 * S D)$} \\
\hline & & 1st_5 min & 2nd_5 min & 1st_5 min & 2nd_5 min & 1st_5 min & 2nd_5 min & 1st_5 min & 2nd_5 min \\
\hline \multirow[t]{4}{*}{ 1st training camp $(n=15)$} & 1st_30s & $\begin{array}{c}-0.55 \\
(-1.17 \\
0.05)^{\#}\end{array}$ & $\begin{array}{c}-0.56 \\
(-1.18 \\
0.05)^{\#}\end{array}$ & $\begin{array}{c}0.38(0.01 \\
0.68)^{\#}\end{array}$ & $\begin{array}{c}0.23(-0.16 \\
0.58)^{\star}\end{array}$ & 3.04 & 3.39 & $\begin{array}{c}-2.14 \\
(-10.07 \\
5.78)\end{array}$ & $\begin{array}{c}-2.23 \\
(-11.56 \\
7.10)\end{array}$ \\
\hline & 1st_60s & $\begin{array}{c}-0.34 \\
(-0.95 \\
0.26)^{\#}\end{array}$ & $\begin{array}{c}-0.35 \\
(-0.97 \\
0.25)^{\#}\end{array}$ & $\begin{array}{c}0.59(0.23 \\
0.81)^{\dagger}\end{array}$ & $\begin{array}{c}0.44(0.37 \\
0.72)^{\#}\end{array}$ & 2.32 & 2.72 & $\begin{array}{c}-1.28 \\
(-7.67 ; 5.12)\end{array}$ & $\begin{array}{c}-1.36 \\
(-9.15 ; 6.42)\end{array}$ \\
\hline & 2nd_30s & $\begin{array}{c}-0.66 \\
(-1.29 \\
0.05)^{\dagger}\end{array}$ & $\begin{array}{c}-0.66 \\
(-1.29 \\
0.06)^{\dagger}\end{array}$ & $\begin{array}{c}0.40(0.02 \\
0.69)^{\#}\end{array}$ & $\begin{array}{c}0.44(0.05 \\
0.72)^{\#}\end{array}$ & 4.80 & 4.63 & $\begin{array}{c}-3.44 \\
(-13.70 \\
6.83)\end{array}$ & $\begin{array}{c}-3.52 \\
(-13.38 \\
6.33)\end{array}$ \\
\hline & 2nd_60s & $\begin{array}{c}-0.56 \\
(-1.18 \\
0.05)^{\#}\end{array}$ & $\begin{array}{c}-0.57 \\
(-1.19 \\
0.04)^{\#}\end{array}$ & $\begin{array}{c}0.49(0.10 \\
0.75)^{\#}\end{array}$ & $\begin{array}{c}0.53(0.14 \\
0.78)^{\dagger}\end{array}$ & 3.98 & 3.82 & $\begin{array}{c}-2.72 \\
(-11.49 \\
6.05)\end{array}$ & $\begin{array}{c}-2.81 \\
(-11.16 \\
5.54)\end{array}$ \\
\hline \multirow[t]{4}{*}{ 2nd training camp $(n=20)$} & 1st_30s & $\begin{array}{c}-0.45 \\
(-0.98 \\
0.08)^{\#}\end{array}$ & $\begin{array}{c}-0.38(0.90 \\
0.15)^{\#}\end{array}$ & $\begin{array}{c}0.52(0.20 \\
0.74)^{\dagger}\end{array}$ & $\begin{array}{c}0.64(0.35 \\
0.81)^{\dagger}\end{array}$ & 4.57 & 3.95 & $\begin{array}{c}-2.85 \\
(-14.35 \\
8.66)\end{array}$ & $\begin{array}{c}-2.16 \\
(-11.09 \\
6.78)\end{array}$ \\
\hline & 1st_60s & $\begin{array}{c}-0.13 \\
(-0.65 ; 0.39)^{\star}\end{array}$ & $\begin{array}{c}-0.02 \\
(-0.54 ; 0.50)^{\star}\end{array}$ & $\begin{array}{c}0.88(0.76 \\
0.94)^{\ddagger}\end{array}$ & $\begin{array}{c}0.81(0.63 ; \\
0.91)^{\ddagger}\end{array}$ & 2.06 & 2.59 & $\begin{array}{c}-0.80 \\
(-6.44 ; 4.85)\end{array}$ & $\begin{array}{c}-0.11 \\
(-6.61 ; 6.40)\end{array}$ \\
\hline & 2nd_30s & $\begin{array}{c}-0.31 \\
(-0.84 \\
0.21)^{\#}\end{array}$ & $\begin{array}{c}-0.23 \\
(-0.76 \\
0.29)^{\#}\end{array}$ & $\begin{array}{c}0.76(0.53 \\
0.88)^{\ddagger}\end{array}$ & $\begin{array}{c}0.51(0.18 \\
0.74)^{\dagger}\end{array}$ & 3.99 & 3.99 & $\begin{array}{c}-2.24 \\
(-11.30 \\
6.82)\end{array}$ & $\begin{array}{c}-1.55 \\
(-14.35 \\
11.25)\end{array}$ \\
\hline & 2nd_60s & $\begin{array}{c}-0.23 \\
(-0.76 \\
0.29)^{\#}\end{array}$ & $\begin{array}{c}-0.17 \\
(-0.67 ; 0.38)^{\star}\end{array}$ & $\begin{array}{c}0.82(0.64 \\
0.91)^{\ddagger}\end{array}$ & $\begin{array}{c}0.65(0.37 \\
0.82)^{\dagger}\end{array}$ & 3.32 & 3.32 & $\begin{array}{c}-1.63 \\
(-9.43 ; 6.18)\end{array}$ & $\begin{array}{c}-0.94 \\
(-11.48 \\
9.60)\end{array}$ \\
\hline \multirow[t]{4}{*}{ 3rd training camp $(n=17)$} & 1st_30s & $\begin{array}{c}-0.65 \\
(-1.24 \\
0.08)^{\dagger}\end{array}$ & $\begin{array}{c}-0.87 \\
(-1.48 \\
0.29)^{\dagger}\end{array}$ & $\begin{array}{c}0.58(0.13 \\
0.81)^{\dagger}\end{array}$ & $\begin{array}{c}0.39(-0.01 \\
0.68)^{\#}\end{array}$ & 3.32 & 4.01 & $\begin{array}{c}-3.21 \\
(-10.58 \\
4.16)\end{array}$ & $\begin{array}{c}-4.06 \\
(-12.71 \\
4.58)\end{array}$ \\
\hline & 1st_60s & $\begin{array}{c}-0.32 \\
(-0.89 \\
0.25)^{\#}\end{array}$ & $\begin{array}{c}-0.54 \\
(-1.12 \\
0.03)^{\#}\end{array}$ & $\begin{array}{c}0.81(0.57 \\
0.92)^{\ddagger}\end{array}$ & $\begin{array}{c}0.61(0.23 \\
0.82)^{\dagger}\end{array}$ & 1.96 & 2.81 & $\begin{array}{c}-1.45 \\
(-6.28 ; 3.38)\end{array}$ & $\begin{array}{c}-2.30 \\
(-8.75 ; 4.15)\end{array}$ \\
\hline & 2nd_30s & $\begin{array}{c}-0.34 \\
(-0.91 \\
0.23)^{\#}\end{array}$ & $\begin{array}{c}-0.55 \\
(-1.13 \\
0.02)^{\#}\end{array}$ & $\begin{array}{c}0.69(0.40 \\
0.85)^{\dagger}\end{array}$ & $\begin{array}{c}0.63(0.23 \\
0.83)^{\dagger}\end{array}$ & 2.68 & 2.93 & $\begin{array}{c}-1.59 \\
(-8.48 ; 5.31)\end{array}$ & $\begin{array}{c}-2.44 \\
(-8.86 ; 3.98)\end{array}$ \\
\hline & 2nd_60s & $\begin{array}{c}-0.11 \\
(-0.68 ; 0.45)^{\star}\end{array}$ & $\begin{array}{c}-0.31 \\
(-0.88 \\
0.25)^{\#}\end{array}$ & $\begin{array}{c}0.80(0.59 \\
0.91)^{\ddagger}\end{array}$ & $\begin{array}{c}0.83(0.59 \\
0.93)^{\ddagger}\end{array}$ & 2.13 & 1.96 & $\begin{array}{c}-0.53 \\
(-6.34 ; 5.28)\end{array}$ & $\begin{array}{c}-1.38 \\
(-5.84 ; 3.07)\end{array}$ \\
\hline \multirow[t]{4}{*}{ 4th training camp $(n=14)$} & 1st_30s & $\begin{array}{c}-0.91 \\
(-1.58 \\
0.27)^{\dagger}\end{array}$ & $\begin{array}{c}-0.66 \\
(-1.31 \\
0.03)^{\dagger}\end{array}$ & $\begin{array}{c}0.51(-0.03 \\
0.79)^{\dagger}\end{array}$ & $\begin{array}{c}0.57(0.11 \\
0.82)^{\dagger}\end{array}$ & 2.86 & 2.68 & $\begin{array}{c}-3.33 \\
(-8.59 ; 1.93)\end{array}$ & $\begin{array}{c}-2.50 \\
(-8.23 ; 3.22)\end{array}$ \\
\hline & 1st_60s & $\begin{array}{c}-0.54 \\
(-1.18 \\
0.09)^{\#}\end{array}$ & $\begin{array}{c}-0.31 \\
(-0.94 \\
0.31)^{\#}\end{array}$ & $\begin{array}{c}0.71(0.23 \\
0.89)^{\ddagger}\end{array}$ & $\begin{array}{c}0.77(0.49 \\
0.90)^{\ddagger}\end{array}$ & 2.33 & 2.07 & $\begin{array}{c}-2.05 \\
(-6.51 ; 2.41)\end{array}$ & $\begin{array}{c}-1.23 \\
(-6.00 ; 3.55)\end{array}$ \\
\hline & 2nd_30s & $\begin{array}{c}-0.67 \\
(-1.32 \\
0.04)^{\dagger}\end{array}$ & $\begin{array}{c}-0.51 \\
(-1.16 \\
0.11)^{\#}\end{array}$ & $\begin{array}{c}0.23(-0.14 \\
0.58)^{\star}\end{array}$ & $\begin{array}{c}0.45(0.06 \\
0.74)^{\dagger}\end{array}$ & 6.33 & 5.35 & $\begin{array}{c}-3.79 \\
(-16.82 \\
9.24)\end{array}$ & $\begin{array}{c}-2.96 \\
(-13.98 \\
8.06)\end{array}$ \\
\hline & 2nd_60s & $\begin{array}{c}-0.47 \\
(-1.12 \\
0.15)^{\#}\end{array}$ & $\begin{array}{c}-0.27 \\
(-0.90 \\
0.35)^{\#}\end{array}$ & $\begin{array}{c}0.51(0.12 \\
0.77)^{\dagger}\end{array}$ & $\begin{array}{c}0.76(0.49 \\
0.90)^{\ddagger}\end{array}$ & 3.33 & 2.33 & $\begin{array}{c}-1.95 \\
(-9.31 ; 5.42)\end{array}$ & $\begin{array}{c}-1.12 \\
(-6.46 ; 4.22)\end{array}$ \\
\hline
\end{tabular}

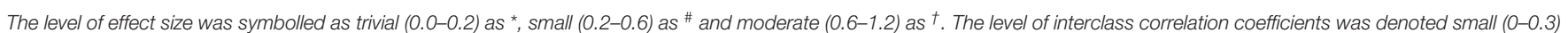

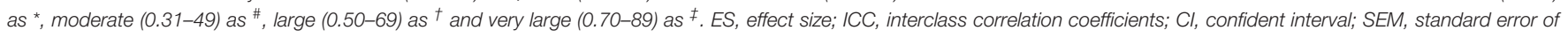
measurement; $S D$, standard deviation; $n$, numbers.

correlations in all correlations analyses except for a very high correlation between the 2nd_5 5 min and 1 st_ $30 \mathrm{~s}(0.83, p<$ $0.001)$, and 2nd_5 min and 1st_60s $(0.88, p<0.001)$. In Figure 2, the results of LnRMSSD $_{\mathrm{cv}}$ demonstrate high (0.59-0.77, $p<0.001)$ to very high $(0.72-0.83, p<0.001)$ correlations in all comparisons.

In Figure 3, the results of LnRMSSD mean $_{\text {show excellent }}$ limits of agreement in all time segment comparisons. The 

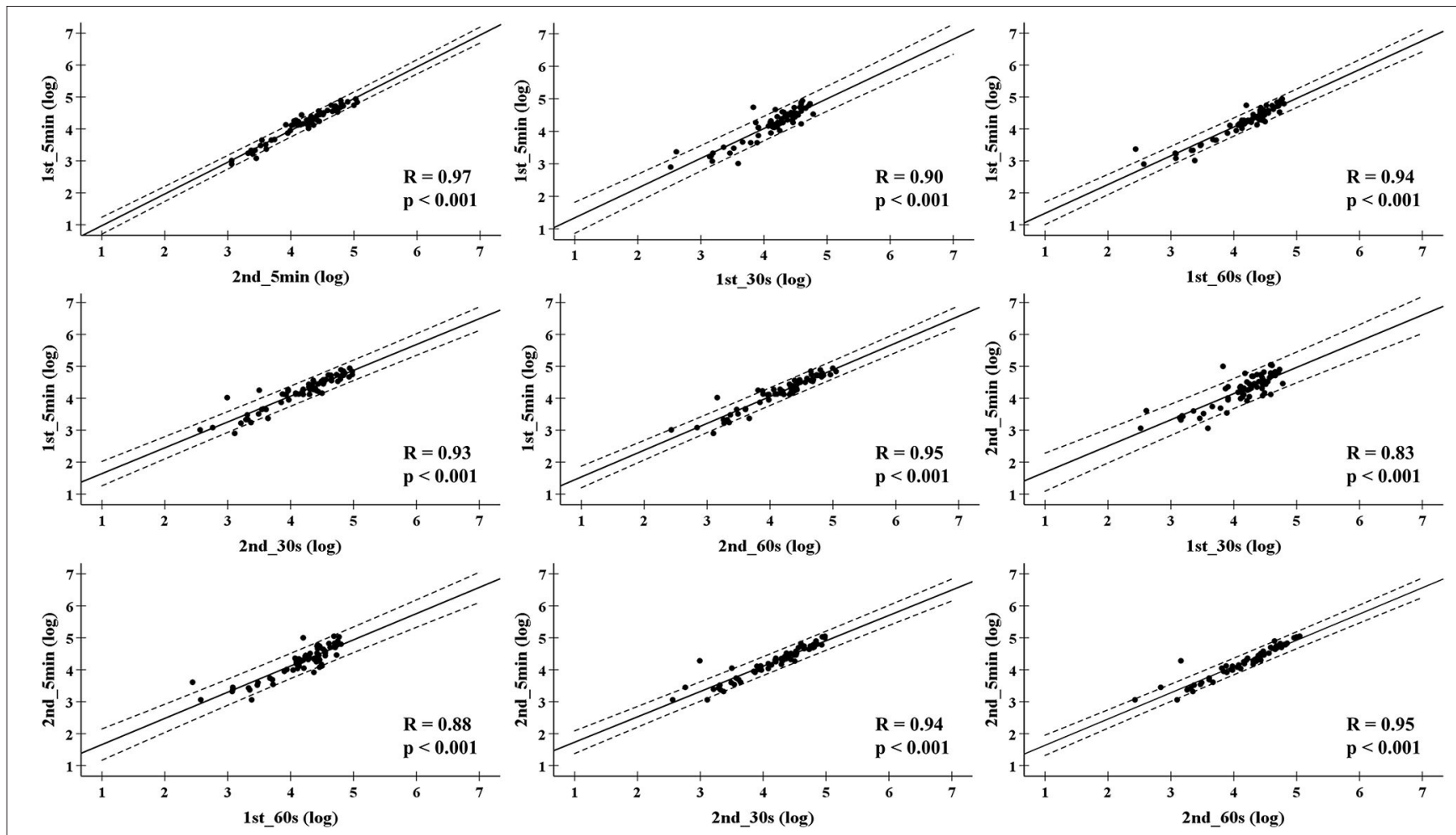

FIGURE 1 | Pearson correlation coefficient for the mean of natural logarithm of the root mean square differences between adjacent normal R-R intervals in all time segments during four overseas training camps. 1st_5 min = The first 5-min LnRMSSD; $1 \mathrm{st} \_30 \mathrm{~s}=$ The first $30 \mathrm{~s}$ LnRMSSD; $1 \mathrm{st} \_60 \mathrm{~s}=$ The first 60 s LnRMSSD; 2nd_5 min = The 6-10 min LnRMSSD; 2nd_30 s = The secondary 30 s LnRMSSD; 2nd_60 s = The secondary 60 s LnRMSSD.

smallest mean difference and the upper and lower limits of agreements was found in the 1 st_5 min and 2nd_5 min comparison (difference $=-0.05,+1.96 \mathrm{~s}=-0.29,-1.96 \mathrm{~s}=$ 0.19). In Figure 4, the results of LnRMSSD $_{\mathrm{cv}}$ demonstrate a wide range of limits of agreement in all time segment comparisons.

\section{Comparison of LnRMSSD $D_{\text {mean }}$ and LnRMSSD $_{\text {cv }}$ Among Overseas Training Camps}

The descriptive results of $\mathrm{LnRMSSD}_{\text {mean }}$ and $\mathrm{LnRMSSD}_{\mathrm{cv}}$ for all time segments during four different overseas training camps are presented in Table 4. Collectively, pairwise comparisons showed trivial to small ES in LnRMSSD mean $(0.02 ; 0.41 \mathrm{ES})$ and LnRMSSD $_{\mathrm{cv}}(-0.02 ;-0.49 \mathrm{ES})$ across overseas training camps.

\section{DISCUSSION}

This study is the first to report the reliability and validity of $\mathrm{HRV}_{\text {UST }}$ during a series of overseas training camps in male U20 national team futsal players. The primary findings of the present study revealed that the 30 and $60 \mathrm{~s}$ HRV UST measure during stabilization and post-stabilization periods were valid and acceptable measures for LnRMSSD assessment and can be used as a surrogate for the standard 5-min recording. In addition, the secondary finding in the present study showed trivial to small levels of effect size in all time segments of LnRMSSD $_{\text {mean }}$ and LnRMSSD $_{\mathrm{cv}}$ across overseas training camps.

\section{Agreement and Reliability of Ultra-Short-Term Heart Rate Variability}

The present study attempted to investigate the agreement of HRV UST assessment for cardiac-autonomic adaptation during and after the stabilization process. The results of LnRMSSD mean demonstrated trivial to small ES, very large to nearly perfect ICC, and narrow range of SEM (0.06-0.31) when all time segments of HRV UST were compared to the 1st_5 min and 2nd_5 min HRV. Furthermore, the magnitude of the correlation coefficients was nearly perfect when the 1st_5 min was compared to all the time segments $(r=0.90-0.97)$. In terms of agreement of HRV UST, we found that there was excellent acceptance in all time segments. Indeed, perfect agreement was found between the 1st_5 min and 2nd_5 min HRV comparison (narrow risk of bias and limits of agreement). These findings indicate an absence of stabilization prior to $\mathrm{HRV}_{\text {UST }}$ measurement is acceptable for the accuracy of HRV measurement during overseas training camps. Our laboratory recently reported acceptance of 30-s HRV UST of LnRMSSD mean measure during short-term training camps in young adult futsal players (Chen et al., 2020). Nakamura et al. (2020) compared the limits of agreement between a standard $10 \mathrm{~min}$ LnRMSSD (5-min stabilization and 5-min HRV record) and 

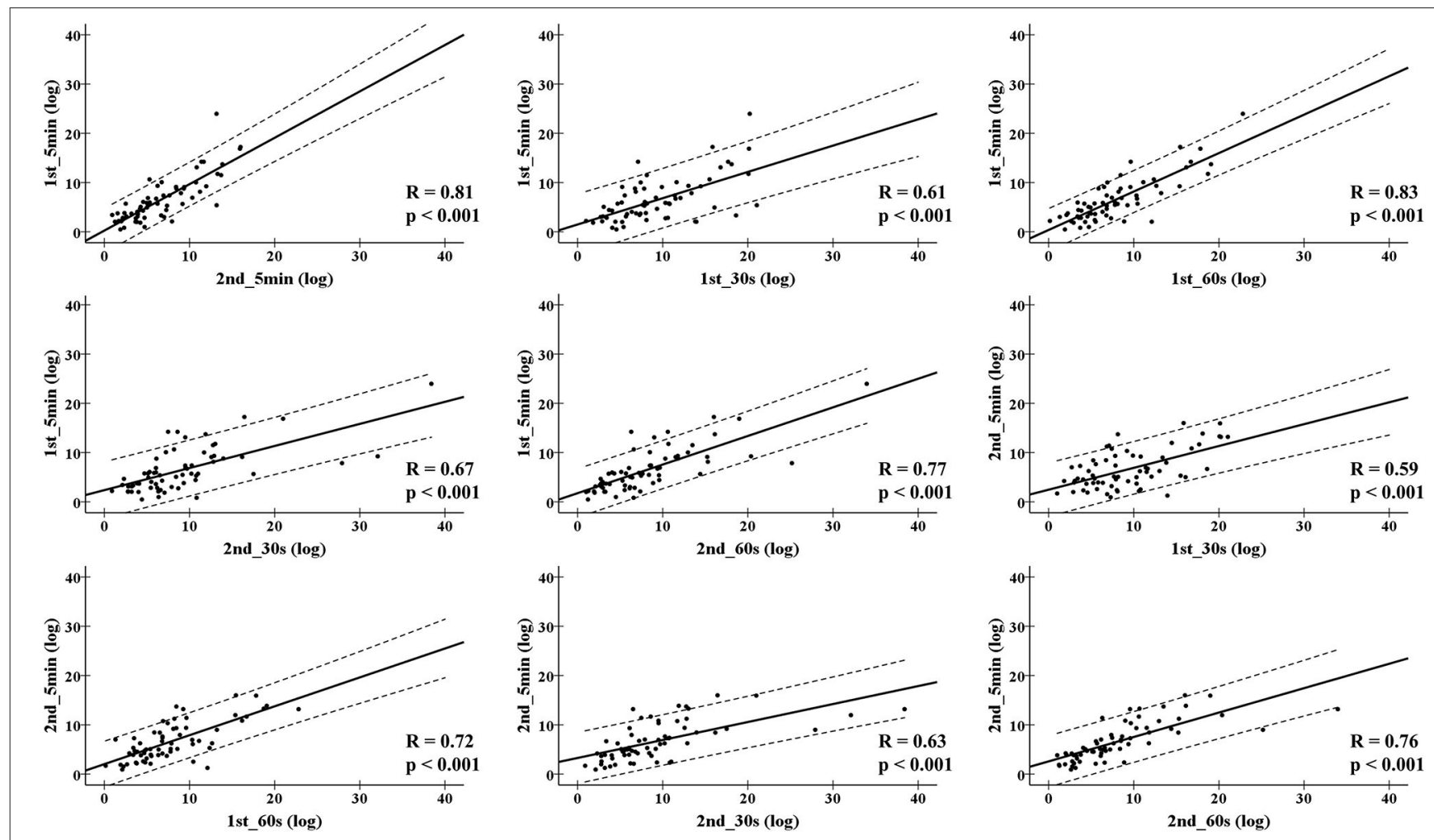

FIGURE 2 | Pearson correlation coefficient for the coefficient of variation of natural logarithm of the root mean square differences between adjacent normal R-R intervals in all time segments during four overseas training camps. 1st_5 min = The first 5-min LnRMSSD; 1st_30 s = The first $30 \mathrm{~s}$ LnRMSSD; 1 st_60 s = The first 60 s LnRMSSD; 2nd_5 min = The 6-10 min LnRMSSD; 2nd_30 s = The secondary 30 s LnRMSSD; 2nd_60 s = The secondary 60 s LnRMSSD.

LnRMSSD UST (1-2 min record followed by 1-min stabilization) in 11 male futsal players before and after 4-weeks of preseason training. Nakamura's et al. study showed meaningful changes in HRV UST measures in response to a futsal preseason (these results indicate a progressive increase in the vagal activity and reduction of training-induced perturbation of cardiac autonomic homeostasis over the futsal pre-season). Excellent agreement and reliability of HRV UST after $1 \mathrm{~min}$ stabilization has also been reported in athletic populations (Esco and Flatt, 2014; Nakamura et al., 2015; Flatt and Esco, 2016; Pereira et al., 2016). Our observation further supports the notion of implementing HRV UST recording by measuring

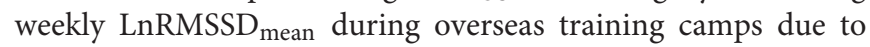
the very high and nearly perfect correlation coefficient and a narrow range of limits of agreement during all time segments. Indeed, all players in our study were familiarized with the procedure of HRV measurement in order to increase the accuracy of recording. Collectively, our findings demonstrate consistent excellent agreement of HRV UST of weekly LnRMSSD $_{\text {mean }}$ during overseas training camps without the conventional 5-min postural stabilization period recommended when LnRMSSD is used for HRV recording.

The results of LnRMSSD $_{\mathrm{cv}}$ demonstrated trivial to large ES, small to very large ICC when $\mathrm{HRV}_{\text {UST }}$ parameters were used to compare the $1 \mathrm{st} \_5 \mathrm{~min}$ and 2 nd_5 min HRV. In addition, high to very high correlations and a wide range of limits of agreement in all time segments of LnRMSSD $_{\mathrm{cv}}$ comparisons were identified (Figures 2, 4). It seems that the shortened $\operatorname{LnRMSSD}_{\mathrm{cv}}$ recording less than 1 min may increase the bias of measurement. Our laboratory recently reported that $\mathrm{HRV}_{\mathrm{UST}}$ of $\mathrm{LnRMSSD}_{\mathrm{cv}}$ could not be used as a surrogate of $5 \mathrm{~min}$ standard HRV records during short-term training camps due to inaccuracy of measures (Chen et al., 2020). It is important to note that $\operatorname{LnRMSSD}_{\mathrm{cv}}$ increases in association with perception of fatigue and reduce of physical performance (Flatt et al., 2017a). Previous studies have demonstrated that 1-min $\operatorname{LnRMSSD}_{\mathrm{cv}}$ measure after 1min postural stabilization is sensitive to physical adaptation in response to periodization of training loads in women soccer players (Flatt and Esco, 2015), rugby seven players (Flatt and Howells, 2019), sprint swimmers (Flatt et al., 2017b), and futsal players (Nakamura et al., 2020). Interestingly, in our study, 60 s HRV $_{\text {UST }}$ of LnRMSSD $_{\mathrm{cv}}$ demonstrated better ICC values, magnitude of correlation coefficient, and agreement of measures than $30 \mathrm{~s} \mathrm{HRV}_{\text {UST }}$ of LnRMSSD $_{\mathrm{cv}}$ despite stabilization or after stabilization period. Nevertheless, cautions should be taken when $\operatorname{LnRMSSD}_{\mathrm{cv}}$ are used to evaluate ANS adaptation during training camps.

\section{Comparisons of LnRMSSD $D_{\text {mean }}$ and LnRMSSD $_{\text {cv }}$ Among Oversea Training Camps}

As demonstrated in Table 4, in comparison with LnRMSSD mean among overseas training camps, trivial ES was observed in 1st_5 min, 2nd_30s, 2nd_60s, and 2nd_5in time segments. In contrast, trivial to small ES in 1 st_30 s and 1st_60 s time 

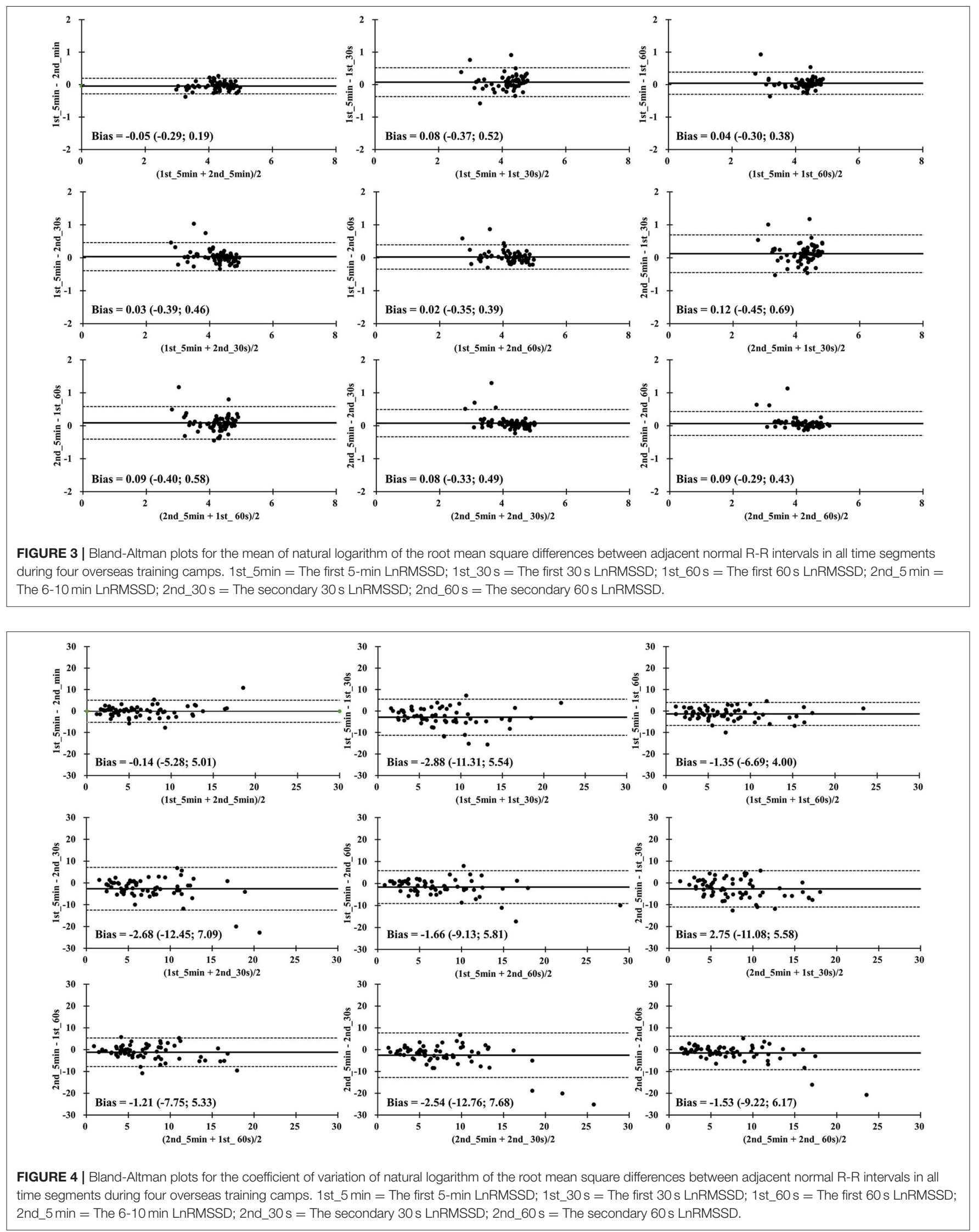
TABLE 4 | Natural logarithm of the root mean square differences between adjacent normal R-R intervals (mean and coefficient of variation values) in first 30 s, first 60 s, and 5 min time segments during stabilization vs after stabilization in four overseas training camps.

\begin{tabular}{|c|c|c|c|c|c|c|c|c|c|c|}
\hline & 1st TC & 2nd TC & 3rd TC & 4th TC & 1 vs. 2 ES & 1 vs. 3 ES & 1 vs. 4 ES & 2 vs. 3 ES & 2 vs. 4 ES & 3 vs. 4 ES \\
\hline \multicolumn{11}{|c|}{ LnRMSSDmean } \\
\hline 1st_5min & $4.21 \pm 0.53$ & $4.19 \pm 0.57$ & $4.22 \pm 0.52$ & $4.20 \pm 0.40$ & $\begin{array}{c}0.04 \\
(-0.53 ; 0.60)\end{array}$ & $\begin{array}{c}-0.02 \\
(-0.60 ; 0.56)\end{array}$ & $\begin{array}{c}0.02 \\
(-0.59 ; 0.63)\end{array}$ & $\begin{array}{c}-0.05 \\
(-0.60 ; 0.49)\end{array}$ & $\begin{array}{c}-0.02 \\
(-0.59 ; 0.55)\end{array}$ & $\begin{array}{c}0.04 \\
(-0.55 ; 0.64)\end{array}$ \\
\hline 1st_30s & $4.27 \pm 0.39$ & $4.04 \pm 0.64$ & $4.11 \pm 0.45$ & $4.14 \pm 0.40$ & $\begin{array}{c}0.41 \\
(-0.15 ; 0.99)\end{array}$ & $\begin{array}{c}0.37 \\
(-0.21 ; 0.96)\end{array}$ & $\begin{array}{c}0.32 \\
(-0.29 ; 0.94)\end{array}$ & $\begin{array}{c}-0.12 \\
(-0.67 ; 0.42)\end{array}$ & $\begin{array}{c}-0.18 \\
(-0.75 ; 0.40)\end{array}$ & $\begin{array}{c}-0.07 \\
(-0.66 ; 0.52)\end{array}$ \\
\hline 1st_60s & $4.28 \pm 0.46$ & $4.09 \pm 0.67$ & $4.16 \pm 0.50$ & $4.15 \pm 0.42$ & $\begin{array}{c}0.32 \\
(-0.25 ; 0.89)\end{array}$ & $\begin{array}{c}-0.12 \\
(-0.70 ; 0.47)\end{array}$ & $\begin{array}{c}0.29 \\
(-0.32 ; 0.91)\end{array}$ & $\begin{array}{c}-0.11 \\
(-0.66 ; 0.43)\end{array}$ & $\begin{array}{c}-0.10 \\
(-0.68 ; 0.47)\end{array}$ & $\begin{array}{c}0.02 \\
(-0.57 ; 0.62)\end{array}$ \\
\hline 2nd_5 min & $4.23 \pm 0.54$ & $4.24 \pm 0.57$ & $4.32 \pm 0.45$ & $4.21 \pm 0.39$ & $\begin{array}{c}-0.02 \\
(-0.58 ; 0.54)\end{array}$ & $\begin{array}{c}-0.15 \\
(-0.74 ; 0.43)\end{array}$ & $\begin{array}{c}0.04 \\
(-0.57 ; 0.65)\end{array}$ & $\begin{array}{c}-0.15 \\
(-0.70 ; 0.39)\end{array}$ & $\begin{array}{c}0.06 \\
(-0.51 ; 0.63)\end{array}$ & $\begin{array}{c}0.25 \\
(-0.34 ; 0.85)\end{array}$ \\
\hline 2nd_30 s & $4.17 \pm 0.63$ & $4.15 \pm 0.65$ & $4.26 \pm 0.60$ & $4.10 \pm 0.41$ & $\begin{array}{c}0.03 \\
(-0.53 ; 0.59)\end{array}$ & $\begin{array}{c}-0.19 \\
(-0.78 ; 0.39)\end{array}$ & $\begin{array}{c}0.13 \\
(-0.48 ; 0.74)\end{array}$ & $\begin{array}{c}-0.17 \\
(-0.72 ; 0.37)\end{array}$ & $\begin{array}{c}0.09 \\
(-0.49 ; 0.66)\end{array}$ & $\begin{array}{c}0.30 \\
(-0.29 ; 0.90)\end{array}$ \\
\hline 2nd_60s & $4.14 \pm 0.65$ & $4.17 \pm 0.63$ & $4.26 \pm 0.57$ & $4.16 \pm 0.40$ & $\begin{array}{c}-0.05 \\
(-0.61 ; 0.52)\end{array}$ & $\begin{array}{c}-0.19 \\
(-0.78 ; 0.39)\end{array}$ & $\begin{array}{c}-0.04 \\
(-0.65 ; 0.58)\end{array}$ & $\begin{array}{c}-0.15 \\
(-0.69 ; 0.40)\end{array}$ & $\begin{array}{c}0.02 \\
(-0.56 ; 0.59)\end{array}$ & $\begin{array}{c}0.19 \\
(-0.40 ; 0.79)\end{array}$ \\
\hline \multicolumn{11}{|c|}{ LnRMSSDcv } \\
\hline 1st_5min & $7.01 \pm 3.71$ & $5.39 \pm 5.91$ & $7.35 \pm 4.47$ & $6.33 \pm 2.96$ & $\begin{array}{c}0.31 \\
(-0.25 ; 0.88)\end{array}$ & $\begin{array}{c}-0.08 \\
(-0.65 ; 0.50)\end{array}$ & $\begin{array}{c}0.20 \\
(-0.40 ; 0.80)\end{array}$ & $\begin{array}{c}-0.36 \\
(-0.92 ; 0.18)\end{array}$ & $\begin{array}{c}-0.19 \\
(-0.76 ; 0.39)\end{array}$ & $\begin{array}{c}0.26 \\
(-0.34 ; 0.86)\end{array}$ \\
\hline 1st_30s & $9.15 \pm 3.87$ & $8.23 \pm 6.59$ & $10.56 \pm 5.13$ & $9.66 \pm 4.09$ & $\begin{array}{c}0.16 \\
(-0.40 ; 0.73)\end{array}$ & $\begin{array}{c}-0.30 \\
(-0.89 ; 0.28)\end{array}$ & $\begin{array}{c}-0.13 \\
(-0.74 ; 0.49)\end{array}$ & $\begin{array}{c}-0.38 \\
(-0.94 ; 0.16)\end{array}$ & $\begin{array}{c}-0.25 \\
(-0.82 ; 0.33)\end{array}$ & $\begin{array}{c}0.18 \\
(-0.41 ; 0.79)\end{array}$ \\
\hline 1st_60s & $8.29 \pm 3.63$ & $6.18 \pm 5.94$ & $8.80 \pm 4.49$ & $8.38 \pm 4.33$ & $\begin{array}{c}0.41 \\
(-0.16 ; 0.98)\end{array}$ & $\begin{array}{c}-0.12 \\
(-0.71 ; 0.46)\end{array}$ & $\begin{array}{c}-0.02 \\
(-0.64 ; 0.59)\end{array}$ & $\begin{array}{c}-0.49 \\
(-1.07 ; 0.08)\end{array}$ & $\begin{array}{c}-0.40 \\
(-0.99 ; 0.17)\end{array}$ & $\begin{array}{c}0.09 \\
(-0.50 ; 0.69)\end{array}$ \\
\hline 2nd_5 min & $6.92 \pm 3.92$ & $6.08 \pm 4.46$ & $6.50 \pm 3.89$ & $7.15 \pm 3.27$ & $\begin{array}{c}0.19 \\
(-0.37 ; 0.76)\end{array}$ & $\begin{array}{c}0.11 \\
(-0.48 ; 0.69)\end{array}$ & $\begin{array}{c}-0.06 \\
(-0.67 ; 0.55)\end{array}$ & $\begin{array}{c}-0.10 \\
(-0.64 ; 0.44)\end{array}$ & $\begin{array}{c}-0.26 \\
(-0.84 ; 0.31)\end{array}$ & $\begin{array}{c}-0.18 \\
(-0.77 ; 0.42)\end{array}$ \\
\hline 2nd_30s & $10.45 \pm 6.19$ & $7.63 \pm 8.14$ & $8.94 \pm 4.81$ & $10.11 \pm 7.22$ & $\begin{array}{c}0.37 \\
(-0.20 ; 0.96)\end{array}$ & $\begin{array}{c}0.27 \\
(-0.32 ; 0.87)\end{array}$ & $\begin{array}{c}0.05 \\
(-0.56 ; 0.66)\end{array}$ & $\begin{array}{c}-0.19 \\
(-0.74 ; 0.35)\end{array}$ & $\begin{array}{c}-0.31 \\
(-0.89 ; 0.26)\end{array}$ & $\begin{array}{c}-0.19 \\
(-0.79 ; 0.40)\end{array}$ \\
\hline 2nd_60 s & $9.73 \pm 5.58$ & $7.01 \pm 7.84$ & $7.88 \pm 4.76$ & $8.27 \pm 4.75$ & $\begin{array}{c}0.38 \\
(-0.19 ; 0.96)\end{array}$ & $\begin{array}{c}0.35 \\
(-0.18 ; 0.96)\end{array}$ & $\begin{array}{c}0.27 \\
(-0.34 ; 0.89)\end{array}$ & $\begin{array}{c}-0.13 \\
(-0.67 ; 0.41)\end{array}$ & $\begin{array}{c}-0.18 \\
(-0.76 ; 0.39)\end{array}$ & $\begin{array}{c}-0.08 \\
(-0.68 ; 0.51)\end{array}$ \\
\hline
\end{tabular}

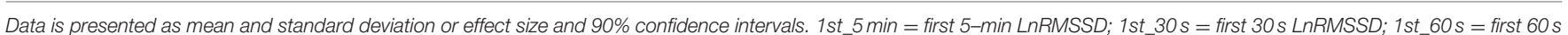
LnRMSSD; 2nd_5min =6-10min LnRMSSD; 2nd_30 s = secondary 30 s LnRMSSD; 2nd_60s = secondary 60s LnRMSSD; TC, training camp; ES, effect size.

segments. Moreover, a wide range of ES between trivial and small levels were identified when LnRMSSD $_{\mathrm{cv}}$ variable were compared across overseas training camps. It is argued that LnRMSSD $_{\text {mean }}$ should be used as a global marker to evaluate the training adaptation of vagal-related activities (Plews et al., 2013b). Whereas, $\operatorname{LnRMSSD}_{\mathrm{cv}}$ is sensitive to detect the daily variation of ANS adaptation to training workloads and psychophysiological conditions (Nakamura et al., 2020). Our laboratory recently reported that $\mathrm{HRV}_{\mathrm{UST}}$ of $\mathrm{LnRMSSD}_{\mathrm{cv}}$ after stabilization period demonstrated large bias and invalid results. In contrast, HRV UST of LnRMSSD mean was valid and reliable to the 5-min standard measure (Chen et al., 2020).

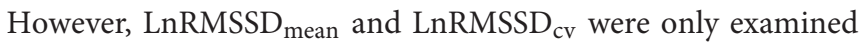
in ultra-short-term and standard time segments with/without stabilization in the present study. Nevertheless, trivial to small ES found in all pairwise comparisons indicated the patterns of

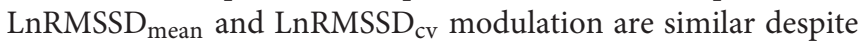
the various objectives of overseas training camps.

\section{Limitations}

The findings in the present study were limited by third factors. Firstly, the number of players vary from camps to camps. Longitudinal adaptation in vagal-related changes in relation to periodization was incomparable in this study Secondly, this study did not compare HRV responses to psychometric and physiological markers of training adaptation. Relationship between HRV UST measures and psychophysiological responses during overseas training camps should be established in future studies. Thirdly, time difference and traveling stress within the fourth overseas training camp may have caused a potential bias in the interpretation of the outcome of the studies. Other logistical issues such as the traveling itinerary, accommodation, accessibility of sports facilities, local transportation, and kit management have a critical effect on players' recovery status.

\section{Practical Implication}

It is understood that time management is a critical issue within training camps. The players require adequate rest time for recovery from the heavy burden of psychophysiological strains due to congested scheduling (i.e., training sessions, matches, meetings, dining, and additional team activities). These factors are considered when additional prerequisites such as medical checks, psychological consultations, rehabilitation, and training load monitoring are facilitated. The findings of this study suggest that HRV monitoring via HRV UST measures within the first minute could be applied to assess vagal-related changes during training camp. 


\section{CONCLUSION}

In conclusion, either the first 30 or 60-s LnRMSSD recordings can be used to evaluate daily cardiac-autonomic functions during overseas training camps in futsal players. The process for stabilization seems to be unnecessary for measuring the morning resting LnRMSSD variable during overseas training camps in young adult futsal players. Consideration to use short HRV UST measures should be addressed due to the discrepancies of LnRMSSD $_{\mathrm{cv}}$ between time segments. In addition, there is a trivial to small variation of weekly $\operatorname{LnRMSSD}_{\text {mean }}$ and $\mathrm{LnRMSSD}_{\mathrm{cv}}$ across different overseas training camps. The observations in our study indicated specific characteristics of HRV modulation in U-20 futsal players during oversea training camps.

\section{DATA AVAILABILITY STATEMENT}

The raw data supporting the conclusions of this article will be made available by the authors, without undue reservation.

\section{ETHICS STATEMENT}

The studies involving human participants were reviewed and approved by the Human Ethics Committee of the University of Taipei. Written informed consent to participate in this study was provided by the participants' legal guardian/next of kin.

\section{REFERENCES}

Bland, J. M., and Altman, D. G. (1986). Statistical methods for assessing agreement between two methods of clinical measurement. Lancet 327, 307-310. doi: 10.1016/S0140-6736(86)90837-8

Buchheit, M. (2014). Monitoring training status with HR measures: do all roads lead to Rome? Front. Physiol. 27:73. doi: 10.3389/fphys.2014.00073

Buchheit, M., Laursen, P. B., and Ahmaidi, S. (2007). Parasympathetic reactivation after repeated sprint exercise. Am. J. Physiol. 293, H133-H141. doi: 10.1152/ajpheart.00062.2007

Castaldo, R., Montesinos, L., Melillo, P., James, C., and Pecchia, L. (2019). Ultrashort term HRV features as surrogates of short term HRV: a case study on mental stress detection in real life. BMC Med. Inform. Decis. Mak. 19:12. doi: 10.1186/s12911-019-0742-y

Chen, Y. S., Clemente, F. M., Bezerra, P., and Lu, Y. X. (2020). Ultra-short-term and short-term heart rate variability recording during training camps and an international tournament in U-20 national futsal players. Int. J. Environ. Res. Public Health 17:775. doi: 10.3390/ijerph17030775

Chen, Y. S., Lu, W. A., Clemente, F. M., Bezerra, J. P., and Kuo, C. D. (2019). Increased parasympathetic activity by foot reflexology massage after repeated sprint test in collegiate football players: a randomised controlled trial. Sports 7:228. doi: $10.3390 /$ sports7110228

Clemente, F. M., Silva, A. F., Sarmento, H., Ramirez-Campillo, R., Chiu, Y. W., Lu, Y. X., et al. (2020). Psychobiological changes during national futsal team training camps and their relationship with training load. Int. J. Environ. Res. Public Health 17:1843. doi: 10.3390/ijerph1 7061843

Esco, M. R., and Flatt, A. A. (2014). Ultra-short-term heart rate variability indexes at rest and post-exercise in athletes: evaluating the agreement with accepted recommendations. J. Sports. Sci. Med. 13, 535-541.

Flatt, A. A., and Esco, M. R. (2015). Smartphone-derived heart-rate variability and training load in a women's soccer team. Int. J. Sports Physiol. Perform 10, 994-1000. doi: 10.1123/ijspp.2014-0556

\section{AUTHOR CONTRIBUTIONS}

Y-SC contributed to the study conceptualization, project administration, investigation, data analysis, methodology, and writing (including reviewing and editing) of the manuscript. JCP and PB contributed to the study conceptualization, data analysis, and writing (including reviewing and editing) of the manuscript. ZC-M contribute to the study data analysis and writing (including reviewing and editing) of the manuscript. C-DK and FMC contributed to the study conceptualization, methodology, supervision, and writing (including reviewing and editing) of the manuscript. All authors contributed to the article and approved the submitted version.

\section{FUNDING}

This study was supported by the grant VN99-14 from the Taipei Veterans General Hospital-National Taiwan University Hospital Joint Research Program, Taipei, Taiwan.

\section{ACKNOWLEDGMENTS}

The authors would like to thanks the head coach Adil Amarante, team staff, and players of the U-20 Chinese Taipei Futsal team who volunteered for this study.

Flatt, A. A., and Esco, M. R. (2016). Heart rate variability stabilization in athletes: towards more convenient data acquisition. Clin. Physiol. Funct. Imaging 36, 331-336. doi: 10.1111/cpf.12233

Flatt, A. A., Esco, M. R., Nakamura, F. Y., and Plews, D. J. (2017a). Interpreting daily heart rate variability changes in collegiate female soccer players. J. Sports. Med. Phys. Fitness. 57, 907-915. doi: 10.23736/S0022-4707.16.06322-2

Flatt, A. A., Hornikel, B., and Esco, M. R. (2017b). Heart rate variability and psychometric responses to overload and tapering in collegiate sprintswimmers. J. Sci. Med. Sport 20, 606-610. doi: 10.1016/j.jsams.2016.10.017

Flatt, A. A., and Howells, D. (2019). Effects of varying training load on heart rate variability and running performance among an olympic rugby sevens team. $J$. Sci. Med. Sport 22, 222-226. doi: 10.1016/j.jsams.2018.07.014

Flatt, A. A., Howells, D., and Williams, S. (2019). Effects of consecutive domestic and international tournaments on heart rate variability in an elite rugby sevens team. J. Sci. Med. Sport 22, 616-621. doi: 10.1016/j.jsams.2018.11.022

Hopkins, W. G., Marshall, S. W., Batterham, A. M., and Hanin, J. (2009). Progressive statistics for studies in sports medicine and exercise science. Med. Sci. Sport Exer. 41, 3-12. doi: 10.1249/MSS.0b013e31818cb278

Krejčí, J., Botek, M., and McKune, A. J. (2018). Stabilization period before capturing an ultra-short vagal index can be shortened to $60 \mathrm{~s}$ in endurance athletes and to $90 \mathrm{~s}$ in university students. PLoS ONE 13:e0205115. doi: 10.1371/journal.pone.0205115

Muñoz-López, A., Nakamura, F., and Naranjo, O. J. (in press). Soccer matches but not training sessions disturb cardiac-autonomic regulation during national soccer team training camps. Res. Q. Exerc. Sport. doi: 10.1080/02701367.2019.1708843. [Epub ahead of print].

Nakamura, F., Soares-Caldeira, L., Laursen, P., Polito, M., Leme, L., and Buchheit, M. (2009). Cardiac autonomic responses to repeated shuttle sprints. Int. J. Sports Med. 30, 808-813. doi: 10.1055/s-0029-1234055

Nakamura, F. Y., Antunes, P., Nunes, C., Costa, J. A., Esco, M. R., and Travassos, B. (2020). Heart rate variability changes from traditional vs. ultra-short-term recordings in relation to preseason training load and performance in futsal players. J. Str. Cond. Res. 34, 2974-2981. doi: 10.1519/JSC.0000000000002910 
Nakamura, F. Y., Flatt, A. A., Pereira, L. A., Ramirez-Campillo, R., Loturco, I., and Esco, M. R. (2015). Ultra-short-term heart rate variability is sensitive to training effects in team sports players. J. Sports. Sci. Med. 14, 602-605.

Pereira, L., Flatt, A., Ramirez-Campillo, R., Loturco, I., and Nakamura, F. (2016). Assessing shortened field-based heart rate variability data acquisition in team-sport athletes. Int. J. Sport. Phys. Perform. 11, 154-158. doi: 10.1123/ijspp.2015-0038

Plews, D. J., Laursen, P. B., Kilding, A. E., and Buchheit, M. (2013a). Evaluating training adaptation with heart-rate measures: a methodological comparison. Int. J. Sport. Phys. Perform. 8, 688-691. doi: 10.1123/ijspp.8.6.688

Plews, D. J., Laursen, P. B., Stanley, J., Kilding, A. E., and Buchheit, M. (2013b). Training adaptation and heart rate variability in elite endurance athletes: opening the door to effective monitoring. Sports Med. 43, 773-781. doi: 10.1007/s40279-013-0071-8

Sandercock, G. R. H., Bromley, P. D., and Brodie, D. A. (2005). Effects of exercise on heart rate variability: inferences from meta-analysis. Med. Sci. Sport. Exer. 37, 433-439. doi: 10.1249/01.MSS.0000155388.39002.9D

Tarvainen, M. P., Niskanen, J. P., Lipponen, J. A., Ranta-aho, P. O., and Karjalainen, P. A. (2014). Kubios HRV - heart rate variability analysis software. Comput. Meth. Prog. Biomed. 113, 210-220. doi: 10.1016/j.cmpb.2013.07.024
Task Force of the European Society of Cardiology and the North American Society of Pacing and Electrophysiology. (1996). Heart rate variability: standards of measurement, physiological interpretation, and clinical use. Circulation 93, 1043-1065. doi: 10.1161/01.CIR. 93.5.1043

Weir, J. P. (2005). Quantifying test-retest reliability using the intraclass correlation coefficient and the SEM. J. Str. Cond. Res. 19, 231-240. doi: 10.1519/00124278-200502000-00038

Conflict of Interest: The authors declare that the research was conducted in the absence of any commercial or financial relationships that could be construed as a potential conflict of interest.

Copyright () 2021 Chen, Pagaduan, Bezerra, Crowley-McHattan, Kuo and Clemente. This is an open-access article distributed under the terms of the Creative Commons Attribution License (CC BY). The use, distribution or reproduction in other forums is permitted, provided the original author(s) and the copyright owner(s) are credited and that the original publication in this journal is cited, in accordance with accepted academic practice. No use, distribution or reproduction is permitted which does not comply with these terms. 